\title{
Development and Preclinical Testing of a Miniaturized Implantable Ventricular Assist Device
}

\author{
A. Arndt*, K. Graichen*, J. Müller*, L. Erd†, H. Krambeck*, M. Göllner* and P. Nüsser* \\ *Berlin Heart GmbH, Berlin, Germany, email: arndt@berlinheart.de \\ †Magnetics Engineering, Berlin, Germany. Email: erd@magnetics-engineering.de
}

\section{Introduction}

Miniaturization of implantable long-term ventricular assist devices promises to decrease the perioperative risk and broaden the application areas towards both biventricular and paediatric support. However, miniaturization has to be traded-off against increasing blood trauma due to potentially higher shear stress levels.

\section{Methods}

Target dimensions of the pump were defined for partially intraventricular placement within the left and right ventricle, respectively. On the basis of a clinically proven magnetic bearing technology pump miniaturization was done iteratively using CFD for design and optimization of the fluid components and 3D-FEM methods for magnetic components design. Optimization criteria were scalar shear stress, hydrodynamic efficiency, radial and axial bearing stiffness and motor efficiency. Bench test methods included measurement of HQ-curves, efficiency and evaluation of rotor dynamics using Laser Doppler Vibrometry. Blood compatibility testing was done with human blood measuring platelet activation and functional capacity, free haemoglobin levels and von Willebrand multimere damage. An initial acute animal test was performed using an ovine model.

\section{Results}

Pump size reduction compared to clinically available pumps was significant. The extraventricular housing volume is 20 cc. The inlet port can be as short as $15 \mathrm{~mm}$ for right ventricular placement. Measured stiffness values for the bearing guarantee a stable operation throughout the whole operation area. Bearing power consumption is below $1 \mathrm{~W}$. At the design point (9100 rpm, $4.5 \mathrm{l} / \mathrm{min}$ against $80 \mathrm{mmHg}$ ) motor power consumption is $4 \mathrm{~W}$. Haemolysis and platelet damage were not higher compared with existing pumps for operation against systemic pressures and much lower against pulmonary artery pressures. Implantation and performance of the pump in the animal model were as expected with no indication to excess blood damage.

\section{Conclusion}

Pump miniaturization seems possible without sacrificing haemocompatibility. The developed pump prototype can be used as a left and right ventricular assist device in the future. 